\title{
THE DESIGN AND ANALYSIS OF PASSIVE HOMOPOLAR NULL FLUX BEARINGS
}

\author{
By: \\ K.R. Davey \\ A. Filatov \\ R.C. Thompson
}

IEEE Transactions on Magnetics, IEEE Transactions on Magnetics, vol. 41, no. 3, March 2005, pp. 1169-1175

\author{
$P R-366$ \\ Center for Electromechanics \\ The University of Texas at Austin \\ PRC, Mail Code R7000 \\ Austin, TX 78712 \\ (512) 471-4496
}




\title{
The Design and Analysis of Passive Homopolar Null Flux Bearing
}

\author{
Kent R. DAVEY, Alexei FLATOV, Richard C. THOMPSON
}

\begin{abstract}
Electrodynamic Maglev (EDS) systems and passive magnetic bearings (PMB) generate magnetic forces by induction through the movement of permanent or superconducting magnets past conducting coils. Nearly all EDS and PMB's systems involve a combination of null flux coil exposed to changing magnetic fields in the rotation or travel direction. A rather unusual variant which allows for the elimination of discrete coils is realized through the use of field structures which do not vary in the direction of rotation. The design and analysis of this homopolar variant is discussed in this paper. This paper offers the first generalized solution of the governing equations as well as the first combined passive damper / axial support bearing. Actuated brushes during motoring and generating simplify energy exchange when the device is used for flywheel energy storage.
\end{abstract}

Index Terms-- null flux, eddy current, induction, maglev, stability, damping, homopolar, flywheel

\section{INTRODUCTION}

$\mathrm{P}$ owell and Danby [1] were the first to introduce the concept of using null flux coils to get necessary induced forces for stable suspensions. Such a system forms the levitation and guidance system of the world's fastest Maglev system in Yamanachi, Japan [2]. Many variations of this design have been proposed both for EDS Maglev [3,4,5] and PMB applications [6]. Passive non-superconducting bearings are the focus in this article.

It is useful to compare a homopolar null flux bearing with a typical null flux PMB such as that depicted in Fig. 1. The permanent magnets are mounted on the rotating member in an alternating fashion. Fixed stationary coils such as that shown in Fig. 2 are centered over the rotating magnet ring. Each of these coils must be made of multi-stranded fine wire to minimize parasitic eddy loss. When the axis of the magnets and that of the coils are coincident, no net flux links the coil. Any displacement of the two axes results in a restoring force which increases with rotation speed $\Omega$. For the six pole arrangement shown with coils having inductance $L$ and resistance $R$, the restoring force will increase as

Manuscript received November 4, 2004. This work was supported in part by NASA and Calnetix under contract \#UTA04-30K.

K.R. Davey and R.C. Thompson are with the Center for Electromechanics, The University of Austin, 1 University Station R7000, Austin, TX 78759, U.S.A. (telephone 512-471-4496, email r.davey, r.thompson @ mail. utexas.edu.

A. Filatov is with Calnetix, 12880 Moore St., Cerritos, CA 90703 (telephone 562-293-1660, email afilatov@calnetix.com.

$$
\frac{2}{\pi} \tan ^{-1}\left(\frac{3 \Omega L}{R}\right) \text { [4]. }
$$

The geometry is stable radially, and to some extent also axially. The stationary coils in Fig. 2 are shown as simple rectangular coils. Although there is some axial restoring force, it is typically small enough to demand a supplemental axial force such as that supplied by the " $U$ " shaped structure at the outer radius. This supplemental axial support structure is unstable radially; its radial destabilizing force must be smaller than the radial restoring force supplied by the primary radial bearing.

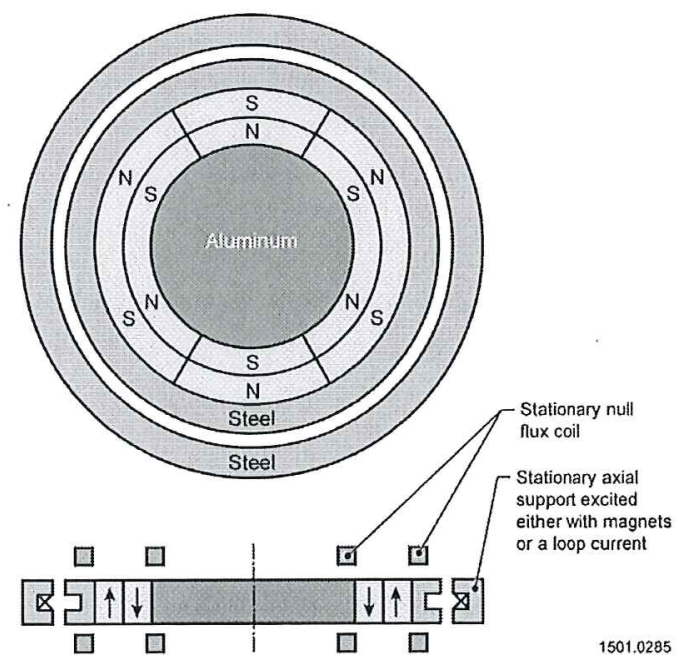

Fig. 1. Typical null flux bearing geometry.

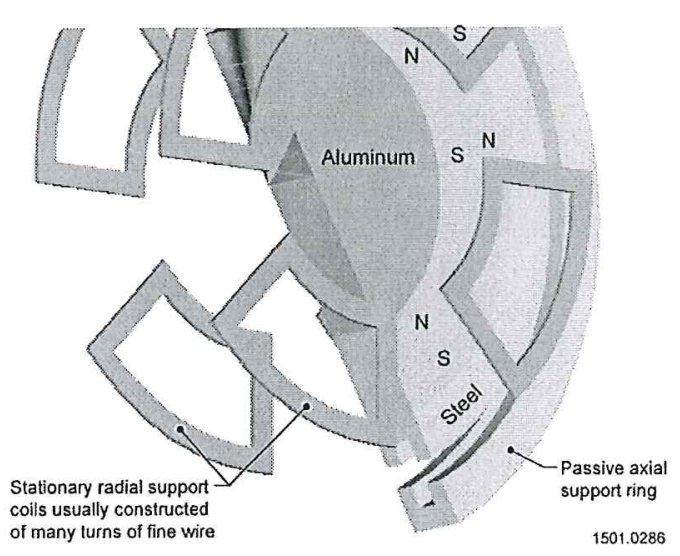

Fig. 2. Three dimensional rendering of a typical PMB. 
Supplemental axial support can be eliminated if the simple rectangular coil is replaced by the more complicated coil shown in Fig. 3. Cross-connecting the upper and lower coils allows for a separate current path which reacts exclusively to axial movement of the magnets with respect to the coils. The primary radial bearing support remains unchanged.

Paul Basore was among the first to work on passive homopolar bearings; this type of bearing has been more recently studied by Filatov. A typical homopolar PMB such as that shown in Fig. 4 shows the magnets displaced to the stationary structure. Discrete null flux coils can be replaced with continuous media such as an aluminum disk. When the axis of the aluminum disk becomes displaced with respect to the stationary magnet axis, the atoms in the aluminum begin experiencing a time changing magnetic field. The resulting induced currents generate forces in an attempt to re-center the disk. The power dissipation resulting from these currents is commensurate with a torque opposing the rotation of the disk. The azimuthal force acts on a moment arm dictated by the displacement distance of the magnetic axis from the coil axis. As will be demonstrated forthwith, this tangential force results in a whirl instability of the disk axis. One way to suppress this instability passively is to introduce a damping agent, such as a magnet ring affixed to the rotating disk driving flux into a stationary aluminum ring.

The advantages of a homopolar null flux bearing over a conventional null flux bearing are as follows:

- Discrete coils are not required, thus allowing for more fabrication options.

- More flexibility is gained in the field assembly. If the magnet assembly is off radially from the axis of the coils, the system will simply seek a new equilibrium axis. In fact, the field can be quite variable in the radial direction. This advantage is offset by the requirement that the field in the azimuthal direction must be constant.

- The parasitic field loss to the encasing structure is virtually eliminated.

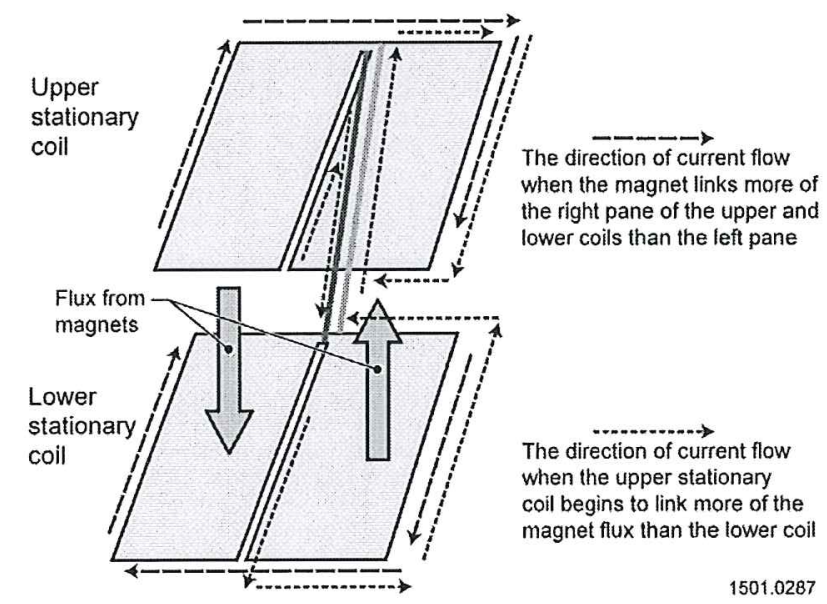

Fig. 3. More complicate null flux coil supplying axial and radial support.
- The primary radial bearing field can serve the dual purpose of the homopolar motor -generator field if intermittent contact with a filament brush can be tolerated.

The disadvantages of the null flux bearing are as follows:

- Non-contact energy extraction is not possible without a separate motor - generator set. The changing magnetic field of a conventional null flux bearing can often serve this dual role.

- The axial restoring force must always be a fraction of the maximum radial restoring force.

This paper outlines the design paradigm for a passive homopolar magnetic bearing. It consists of the following:

- Computing the radial and azimuthal (tangential) restoring forces

- Analyzing the steady state thermal temperature sustainable in a 0.1 torr vacuum

- Determining the necessary damping requirements for stability

- Designing the damper / axial support bearing

- Verifying radial and axial stability

\section{RADIAL AND TANGENTIAL FORCES}

Optimization analysis is employed to determine the permanent magnet geometry to maximize the radial restoring force/ weight ratio. For rotations near $15,000 \mathrm{rpm}$ and displacements near $0.3175 \mathrm{~cm}$ (0.125 in.), the optimized magnet is shown in Fig. 5. A two dimensional field analysis was performed by assigning a lateral velocity to the aluminum shown in Fig. 5. For a displacement distance $\Delta$ of the flywheel axis with respect to the magnet axis, and a rotation speed of $\Omega$ $\mathrm{rpm}$, the equivalent lateral velocity is

$$
v=2 \frac{\Omega}{60} \Delta .
$$

A $15,000 \mathrm{rpm}$ revolution rotation with $D=3.17 \mathrm{~mm}(0.125$ in.) corresponds to $v=1.587 \mathrm{~m} / \mathrm{s}$.

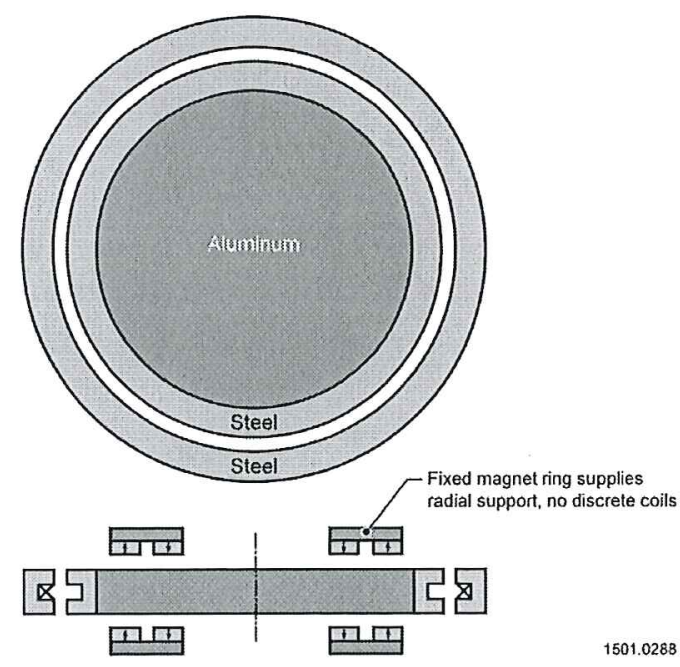

Fig. 4. Homopolar null flux bearing geometry. 


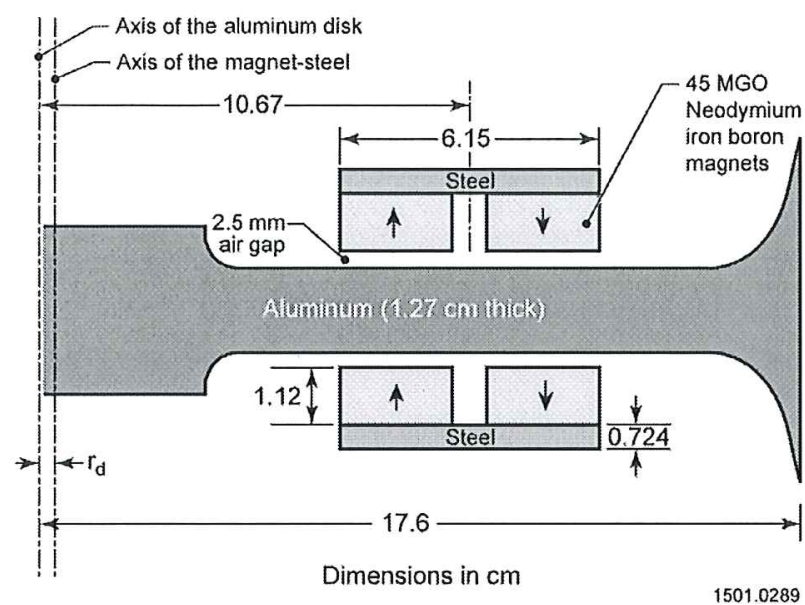

Fig. 5. Ideal magnet and steel configuration when the equivalent velocity is reduced to $1.7 \mathrm{~m} / \mathrm{s}$.

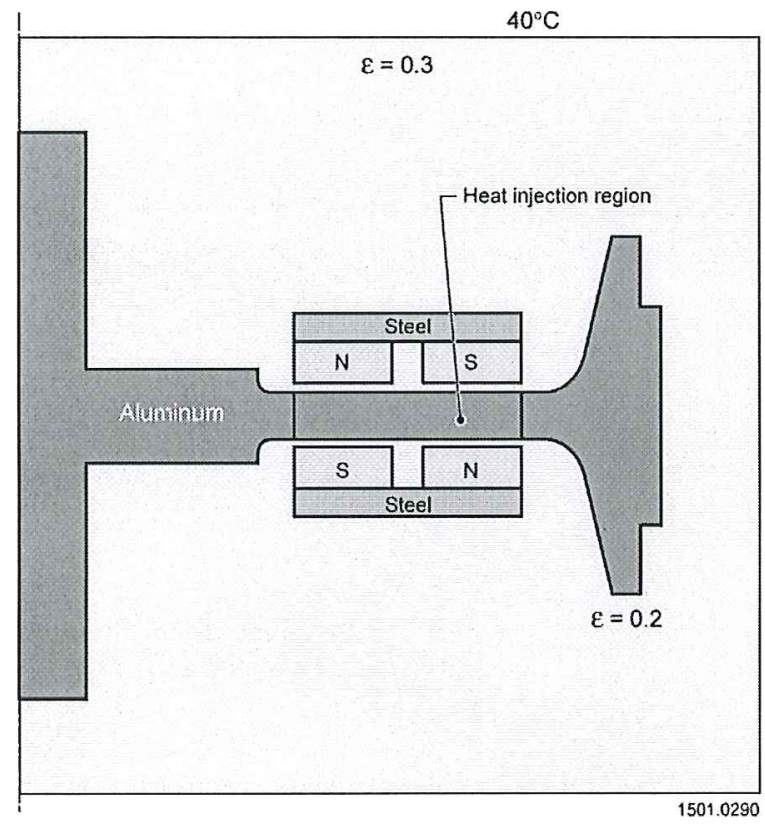

Fig. 6. Thermal analysis setup geometry.

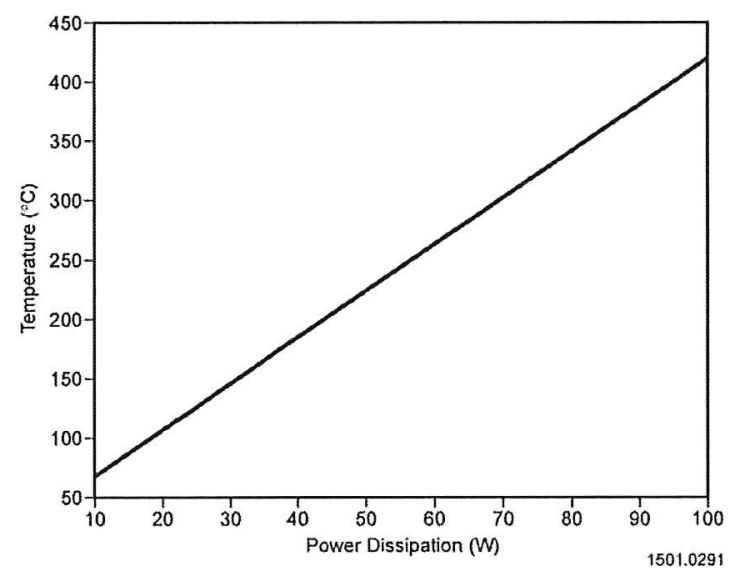

Fig. 7. Temperature on the aluminum versus power dissipated due to eddy currents.
TABLE I.

FORCES AND LOSSES ON THE ALUMINUM DISK FOR VARIOUS ROTATION SPEEDS, ASSUMING A $3.175 \mathrm{MM}(0.125 \mathrm{IN}$.) OFFSET AXIS DISPLACEMENT.

\begin{tabular}{rrcr}
\hline \hline $\begin{array}{r}\text { Rotation } \\
(\mathrm{RPM})\end{array}$ & $\mathrm{Fx}(\mathrm{N})$ & Loss (W) & Ftan (N) \\
\hline 2,143 & 476 & 107 & 150.2 \\
4,286 & 945 & 425 & 298.2 \\
6,429 & 1,388 & 942 & 440.7 \\
8,571 & 1,815 & 1,645 & 577.2 \\
10,714 & 2,216 & 2,510 & 704.6 \\
12,857 & 2,586 & 3,518 & 823.0 \\
15,000 & 2,910 & 4,640 & 930.4 \\
\hline \hline
\end{tabular}

In a two dimensional analysis, all forces and losses are computed on a unit depth basis. With a disk, the lateral velocity becomes collinear with the velocity one-fourth of the way around the disk. One estimate of the appropriate depth is the $\pi$ times the mean radius, i.e., one-half the mean circumference as dictated at the magnet mid line, $r m=10.87$ $\mathrm{cm}$ (4.28 in.). The tangential force can be estimated from the power dissipation in the conductor. It should match the drag torque times the rotation speed. In terms of the power loss $P$,

$$
F_{\text {tan }}=\frac{P}{2 \pi r_{d} \Omega / 60}
$$

The radius $r d$ is the offset axis displacement (see Fig. 5). A self adaptive finite element analysis was performed on the magnet assembly to return the results shown in Table I.

\section{TemPerature CONSTRAINTS}

Before considering stability issues, some thermal considerations are warranted. High tip speeds demand operation in a vacuum. Consider the aluminum hub dimensioned as in Fig. 5 within a surrounding $40^{\circ} \mathrm{C}$ containment vessel as depicted in Fig. 6. The emissivity of the aluminum should be close to 0.2 and that for the steel 0.3 . Axysymmetric boundary element analysis is performed to determine the aluminum temperature. No additional heating of the rotor is assumed from rotation, and all the power is injected into the center aluminum region annotated in Fig. 6. Fig. 7 shows that the slope of the temperature growth is quite steep. Cooling occurs only through radiation. The magnets will degrade with temperature; this effect was not added to the analysis.

Using (2), it is possible to get the restoring force and the power loss as a function of axis offset distance $\Delta$. Fig. 8 shows these results when the axis offset distance ranges from 0.051 $\mathrm{mm}$ to $0.51 \mathrm{~mm}$ ( 2 mil to $20 \mathrm{mil}$ ). The abscissa corresponds to $r d$ in Fig. 5. The aluminum is in physical contact with the composite flywheel. Temperatures on these composites should not exceed $140^{\circ} \mathrm{C}$. This would suggest that the allowed mean offset distance of the rotation axis with respect to the magnetic axis cannot exceed $0.2 \mathrm{~mm}$ to $0.25 \mathrm{~mm}(8 \mathrm{mil}-10 \mathrm{mil})$. When the loss from rotation is added, the number will surely 

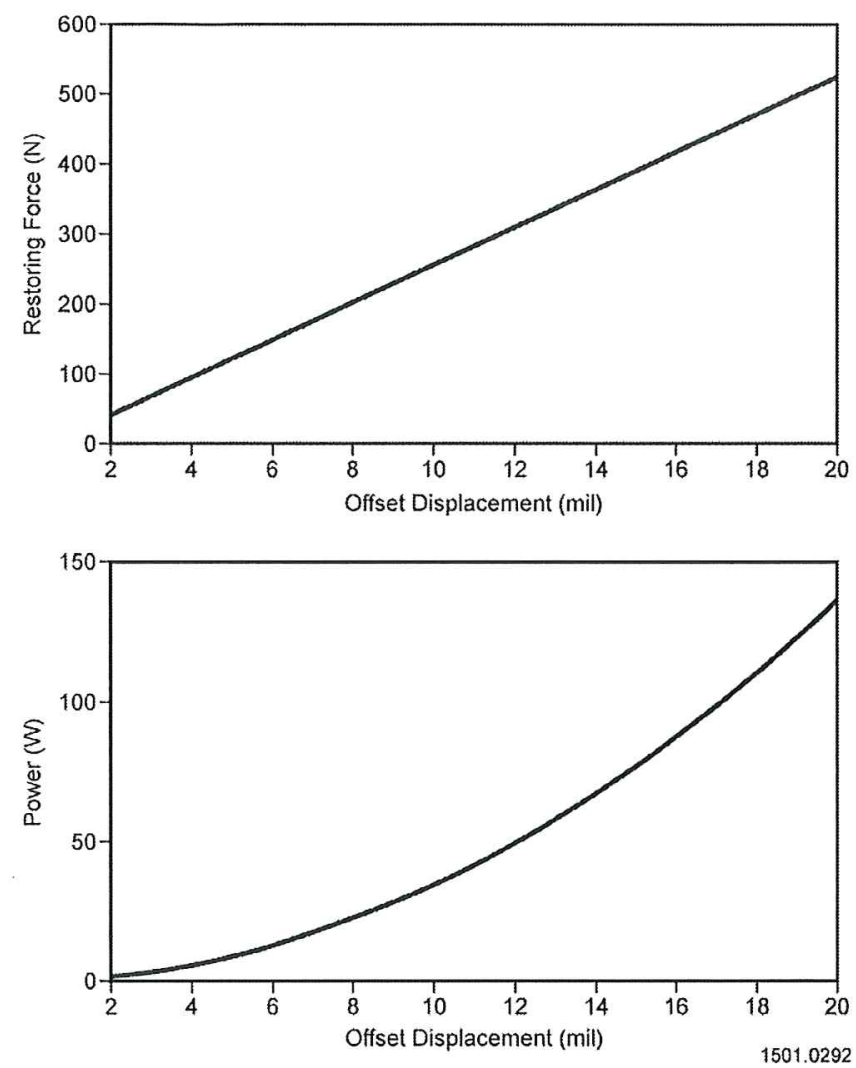

Fig. 8. Restoring force and power dissipation as a function of axial offset distance at 15,000 rpm.

decrease further, perhaps to $0.152 \mathrm{~mm}$ ( 6 mils). Maintaining such a small offset in an environment exposed to vibration would be unreasonable. The bearing would be stable, but it would quickly exceed safety limits.

\section{Stabiltity}

The forces in Table I allow a computation of damper requirements. Any displacement of the bearing axis from the magnetic axis results in both a restoring force co-linear with the displacement and a tangential force normal to the displacement. The equations of motion for the undamped system are

$$
\begin{aligned}
& m \frac{d^{2} x}{d t^{2}}=-k_{1} x+k_{2} y \\
& m \frac{d^{2} y}{d t^{2}}=-k_{1} y-k_{2} x
\end{aligned}
$$

The constants $k_{1}$ and $k_{2}$ are the values registered in columns two and four of Table I. Suppose the bearing begins at position $x_{0}, y_{0}$ with zero velocity. The axis positions $\mathrm{x}$ and $y$ are given by the solution of (4) and (5),

$$
x(t)=\frac{x_{0}}{2} \cos (\omega t)\left(e^{-\beta t}+e^{\beta t}\right)-\frac{y_{0}}{2} \sin (\omega t)\left(e^{-\beta t}-e^{\beta t}\right)
$$

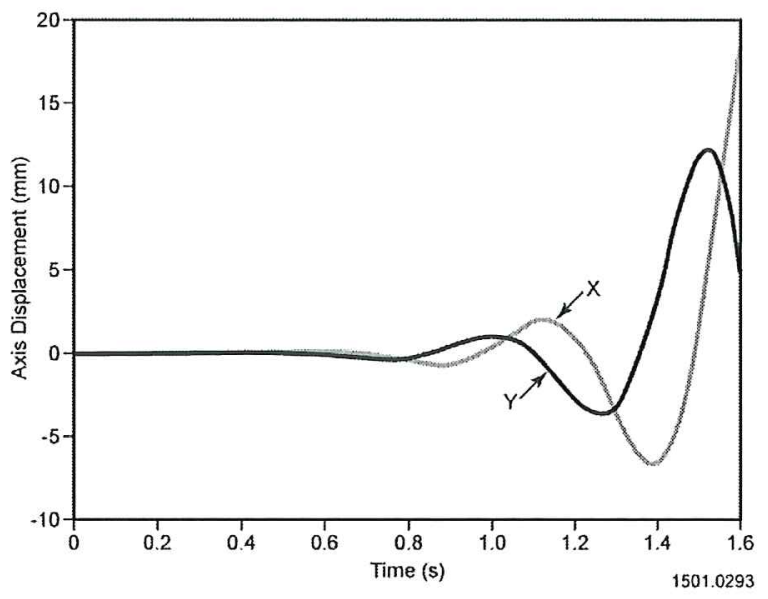

Fig. 9. Displacement of the bearing axis with respect to the magnet axis.

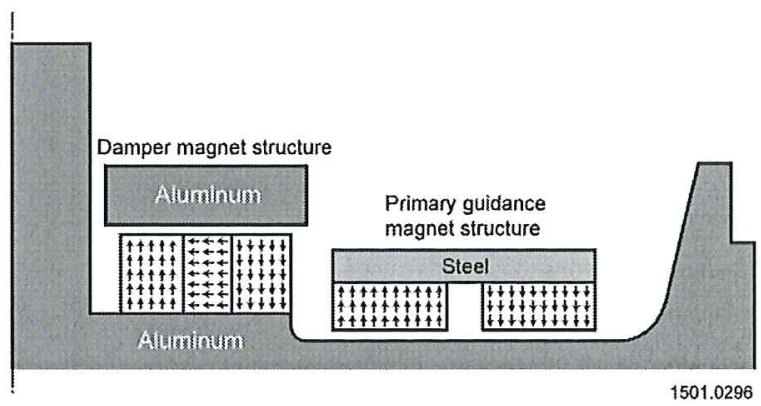

Fig. 10. Damper structure added at a smaller radius.

$$
y(t)=\frac{y_{0}}{2} \cos (\omega t)\left(e^{-\beta t}+e^{\beta t}\right)+\frac{x_{0}}{2} \sin (\omega t)\left(e^{-\beta t}-e^{\beta t}\right)
$$

where

$$
\begin{aligned}
& \beta=\frac{\sqrt{\sqrt{k_{1}^{2}+k_{2}^{2}}}}{\sqrt{m}} \cdot \sin \left(\frac{1}{2} \cdot \tan ^{-1}\left(\frac{k_{1}}{k_{2}}\right)\right) \\
& \omega=\frac{\sqrt{\sqrt{k_{1}^{2}+k_{2}^{2}}}}{\sqrt{m}} \cdot \cos \left(\frac{1}{2} \cdot \tan ^{-1}\left(\frac{k_{1}}{k_{2}}\right)\right)
\end{aligned}
$$

This system is always unstable; a typical growth profile is shown in Fig. 9.

The solution for stabilizing the system passively is to add a damper. Perhaps the most straightforward damper is realized by placing magnets on the rotating member opposite a conducting member as suggested in Fig. 10, essentially the opposite of the existing system. In the existing system, the magnets are stationary and the aluminum rotates. For the damper, the magnets must rotate, and the aluminum remains stationary. This arrangement yields a restoring force proportional to velocity so that the governing equations become 


$$
\begin{aligned}
& m \frac{d^{2} x}{d t^{2}}=-k_{1} x+k_{2} y-D \frac{d x}{d t} \\
& m \frac{d^{2} y}{d t^{2}}=-k_{1} y-k_{2} x-D \frac{d y}{d t}
\end{aligned}
$$

The solution for these equations is

$$
\begin{array}{r}
x(t)=e^{-\left(\frac{D}{2 m} \alpha\right)}\left\{\begin{array}{l}
\cos (\omega t)\left[\left(\alpha \omega-\frac{D}{4 m} \omega\right) y_{0}-\left(\frac{D}{4 m} \alpha+\frac{1}{2}\left(\omega^{2}-\alpha^{2}\right)\right) x_{0}\right] \\
+\sin (\omega t)\left[\left(\alpha \omega-\frac{D}{4 m} \omega\right) x_{0}+\left(\frac{D}{4 m} \alpha+\frac{1}{2}\left(\omega^{2}-\alpha^{2}\right)\right) y_{0}\right]
\end{array}\right\} \\
+e^{-\left(\frac{D}{2 m}+\alpha\right)}\left\{\begin{array}{l}
\cos (\omega t)\left[\left(\alpha \omega+\frac{D}{4 m} \omega\right) y_{0}+\left(\frac{D}{4 m} \alpha-\frac{1}{2}\left(\omega^{2}-\alpha^{2}\right)\right) x_{0}\right] \\
+\sin (\omega t)\left[\left(-\alpha \omega-\frac{D}{4 m} \omega\right) x_{0}+\left(\frac{D}{4 m} \alpha-\frac{1}{2}\left(\omega^{2}-\alpha^{2}\right)\right) y_{0}\right.
\end{array}\right\} \\
y(t)=e^{-\left(\frac{D}{2 m}-\alpha\right)}\left\{\begin{array}{l}
\cos (\omega t)\left[\left(\alpha \omega-\frac{D}{4 m} \omega\right) x_{0}+\left(\frac{D}{4 m} \alpha-\frac{1}{2}\left(\omega^{2}-\alpha^{2}\right)\right) y_{0}\right] \\
+\sin (\omega t)\left[\left(-\alpha \omega-\frac{D}{4 m} \omega\right) y_{0}+\left(-\frac{D}{4 m} \alpha+\frac{1}{2}\left(\omega^{2}-\alpha^{2}\right)\right) x_{0}\right.
\end{array}\right\} \\
+e^{-\left(\frac{D}{2 m}+\alpha\right)}\left\{\begin{array}{l}
\cos (\omega t)\left[\left(-\alpha \omega+\frac{D}{4 m} \omega\right) x_{0}+\left(\frac{D}{4 m} \alpha-\frac{1}{2}\left(\omega^{2}-\alpha^{2}\right)\right) y_{0}\right] \\
+\sin (\omega t)\left[\left(\alpha \omega-\frac{D}{4 m} \omega\right) y_{0}+\left(-\frac{D}{4 m} \alpha-\frac{1}{2}\left(\omega^{2}-\alpha^{2}\right)\right) x_{0}\right]
\end{array}\right\}
\end{array}
$$

where

$$
\alpha=\frac{\sqrt{\sqrt{\left(\frac{D^{2}}{m}-4 k_{1}\right)^{2}+16 k_{2}^{2}}}}{2 \sqrt{m}} \cdot \cos \left(\frac{1}{2} \cdot \tan ^{-1}\left(\frac{4 k_{2}}{\frac{D^{2}}{m}-4 k_{1}}\right)\right)
$$

$$
\omega=\frac{\sqrt{\sqrt{\left(\frac{D^{2}}{m}-4 k_{1}\right)^{2}+16 k_{2}^{2}}}}{2 \sqrt{m}} \cdot \sin \left(\frac{1}{2} \cdot \tan ^{-1}\left(\frac{4 k_{2}}{\frac{D^{2}}{m}-4 k_{1}}\right)\right)
$$

The criteria for stability is that $D /(2 m)>\alpha$. Assume a weight of $15 \mathrm{~kg}$ for the flywheel and bearing. The final row of Table I can be used to determine values for $k_{1}$ and $k_{2}$ at $15,000 \mathrm{rpm}$ to be $0.916 \mathrm{MN} / \mathrm{m}$ and $0.293 \mathrm{MN} / \mathrm{m}$ respectively. A plot of $2 \alpha$ versus damper coefficient $D$ is shown in Fig. 11. Under these conditions, the required damping coefficient must exceed $1.18 \mathrm{kN}-\mathrm{s} / \mathrm{m}$. Since the spring constants grow linearly with speed, the required damping must grow as the square root of speed. Fig. 12 shows the predicted displacements for damping coefficients of $1.1 \mathrm{kN}-\mathrm{s} / \mathrm{m}$ and $1.2 \mathrm{kN}-\mathrm{s} / \mathrm{m}$.

\section{DAMPER AND AXIAL SUPPORT DESIGN}

The requirements for the damper are challenging. The simple damper depicted in Fig. 10 shows the principle. Permanent magnets are added to the rotating structure at a smaller radius. Because they rotate, and the damper structure is homogeneous in the azimuthal direction, no current is induced

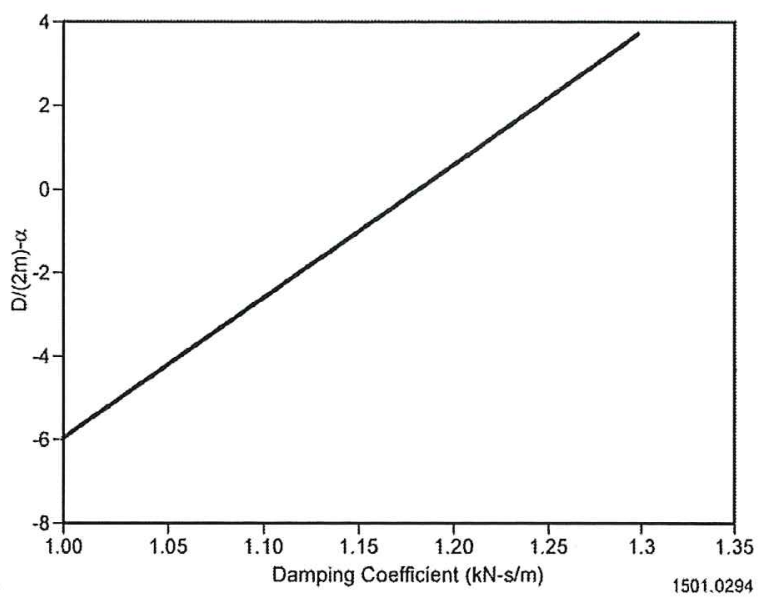

Fig.11. Stability criteria for the magnetic bearing at $15,000 \mathrm{rpm}$.
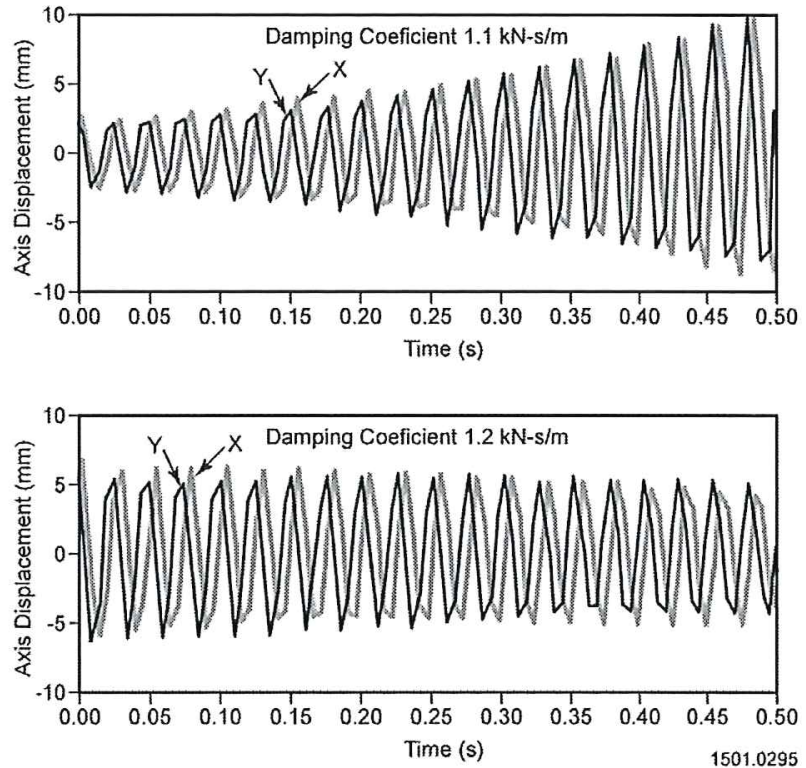

Fig. 12. Profile of axis displacement with two damping coefficients just less than and just greater than that required for stability

in the fixed aluminum block due to rotation, only lateral movement. Unfortunately this simple damper is inadequate for supplying the required damping, supplying only one third of what is required at $15,000 \mathrm{rpm}$.

A better damper design which accomplishes the dual task of axial support is shown in Fig. 13. A Halbach type array is employed to significantly increase the damping force. Fig. 14 shows how the magnetic field compresses between the aluminum and the magnets resulting in a more than adequate damping force of $1.74 \mathrm{kN}-\mathrm{s} / \mathrm{m}$. Replacing this geometry with a copper damper of conductivity of $5.8 \cdot 107 \mathrm{~S} / \mathrm{m}$ yields a damping force of $2.77 \mathrm{kN}-\mathrm{s} / \mathrm{m}$. It should be clear from (12) and (13) that the required damping force scales as the square root of mass. A $50 \mathrm{~kg}$ rotor mass requires a damping force of $2.19 \mathrm{kN}-\mathrm{s} / \mathrm{m}$, so a copper damper would allow quite an increase in rotor mass. 


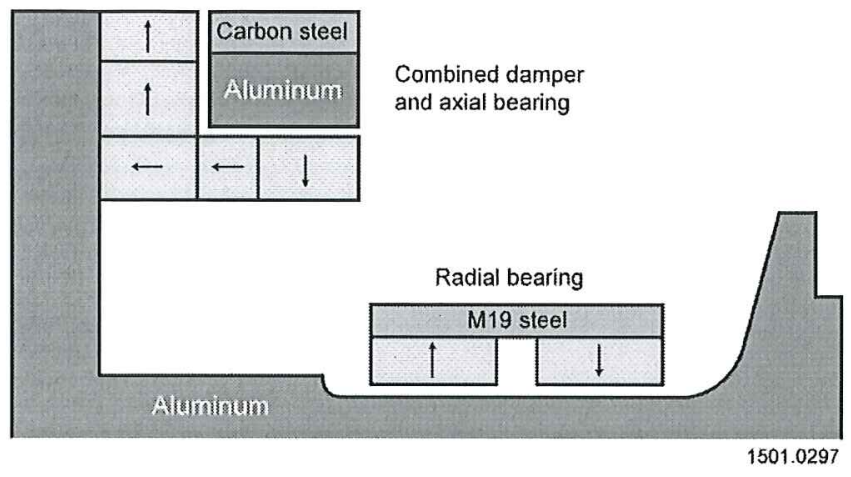

Fig. 13. Complete passive bearing.

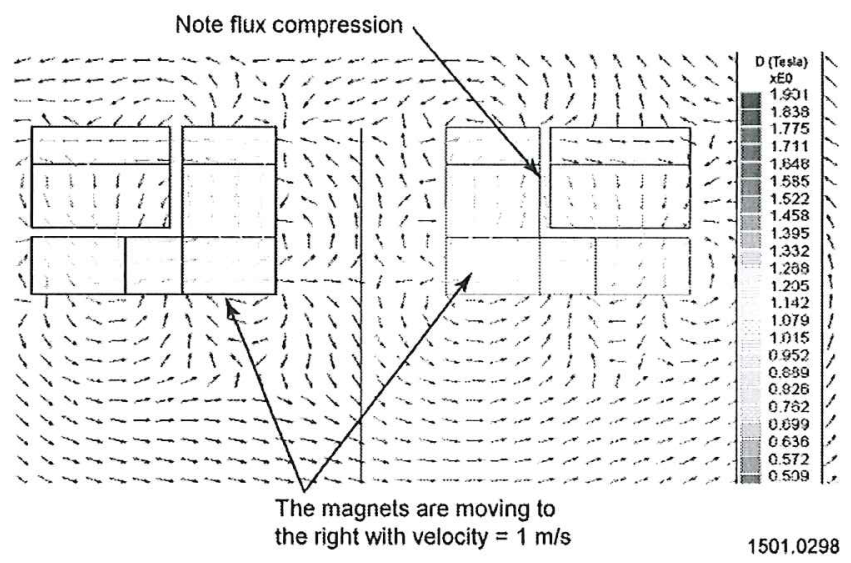

Fig. 14. Field lines resulting from a lateral displacement of the magnets with a velocity of $1 \mathrm{~m} / \mathrm{s}$.

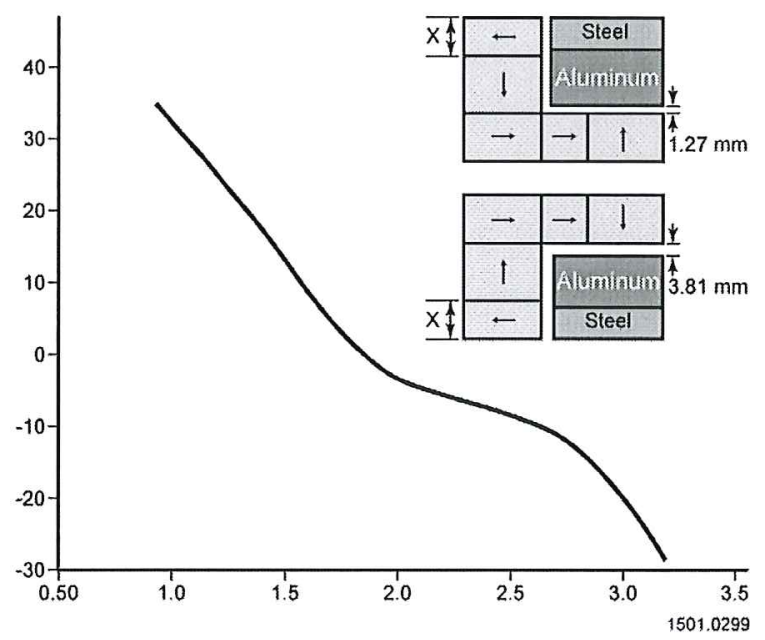

Fig. 15. Axial restoring force variation as a function of the magnet thickness adjacent to the steel. The combined force on the upper and lower steel - aluminum blocks is computed when the upper steel- $/$ aluminum block is $1.27 \mathrm{~mm}$ closer to the magnet ring than the lower steel- aluminum block.

Two potential problems surface with this new structure, axial force stability and destabilizing radial forces.

\section{A. Axial Force Stability}

The thickness of the outer magnet in the damper structure (annotated $x$ in Fig. 15) is critical to axial stability. Fig. 15 shows how the axial restoring force varies with that thickness. If that thickness is kept below $1.8 \mathrm{~cm}$, the axial force is stabilizing. A value of $0.95 \mathrm{~cm}$ is used in this study. This happens because of the strong axial (vertical) force dependence on $\mathrm{X}$. As $\mathrm{x}$ increases, the magnetic field lines have more space to spread out within the steel, and the axial aligning force decreases.

\section{B. Radial Stability}

Although this structure provides the necessary damping force, it also introduces radial destabilizing forces. This destabilizing force is not quite linear, so the equivalent spring constant is position dependent. The problem must be analyzed similar to above. Consider the force developed as the aluminum - steel blocks in quadrants one and two are displaced laterally with respect to the magnet clusters. The destabilizing force and the equivalent position dependent spring constant are plotted in Fig. 16. The primary radial bearing spring constant is computed from Table I. It is velocity dependent and is shown plotted for 15,000 rpm in the lower trace. The stability provided by the primary bearing is more than adequate at this speed. The approximate factor of two margin on stability indicates that the device would require backup bearings below 7,500 rpm. Increasing the radial base gap between magnets and the steel - aluminum block is one obvious method of lowering this stable operating speed. The destabilizing force reduces more rapidly than does the damping coefficient as the radial gap between the steel aluminum block and the magnets increases. Increasing this starting gap from $2.54 \mathrm{~mm}$ to $5.08 \mathrm{~mm}$ reduces the damping coefficient to $83 \%$ of its original value, while reducing the destabilizing magnet force to $46 \%$ of its original value. Such an adjustment would lower the stable operating speed to about $3,400 \mathrm{rpm}$, and still provide adequate damping.

With this modification, the final bearing appears as in Fig. 17. Fig 18 shows a three dimensional rendering of the completed bearing.

\section{MOTOR - GENERATOR}

Although typical motor - generator sets have been shown for this type of bearing [4], a more elegant solution should result from using the homopolar field. Richard Thome has successfully demonstrated the use of filament brushes in homopolar motors; these copper fiber brushes have extremely low down pressures and excellent wear rates. A thirty year life cycle for these brushes is realistic. For many energy applications, motor-generator time is a small percentage of the operation time. The simplest means of coupling power into and out of the bearing is by means of electromagnetic actuated filament brushes as suggested in Fig. 19. When airborne contaminants are problematic for the targeted use environment, a conventional shaft mounted motor generator set is warranted. 


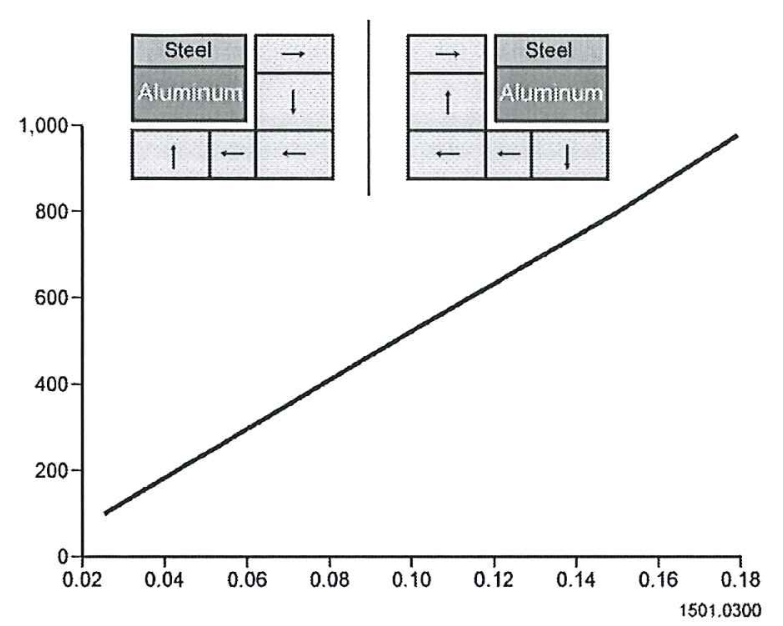

Fig. 16. Destabilizing force variation with displacement position. The aluminum - steel blocks are displaced laterally with respect to the magnet clusters, and the force is computed on the combined steelaluminum blocks.

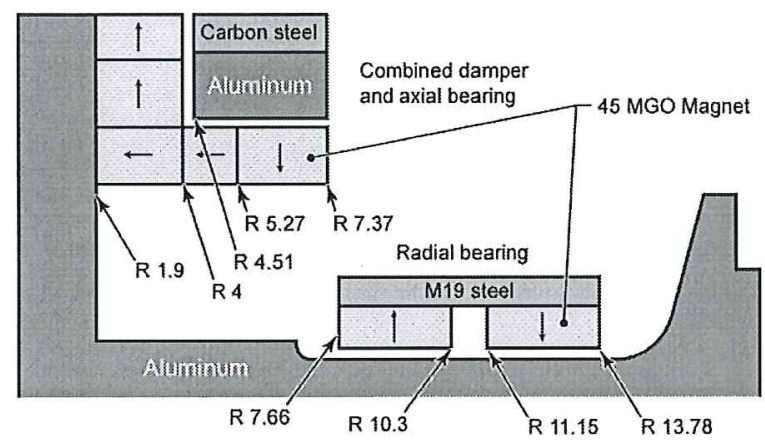

Dimensions in $\mathrm{cm}$

1501.0301

Fig. 17. Full passive bearing with dimensions.

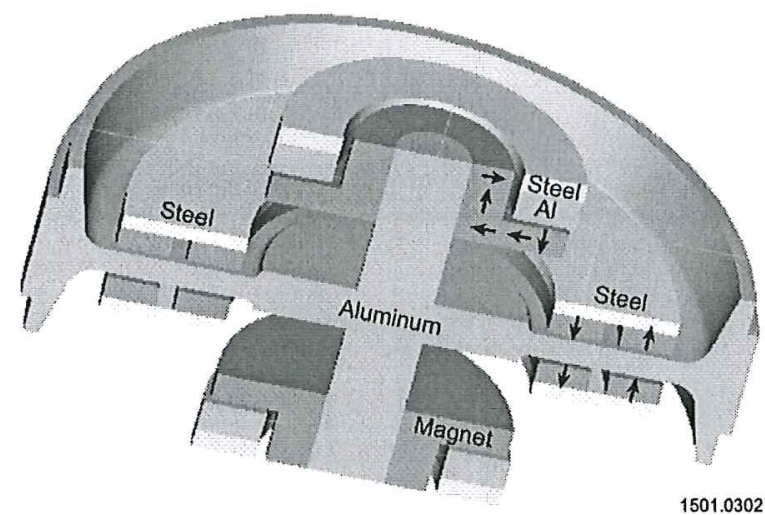

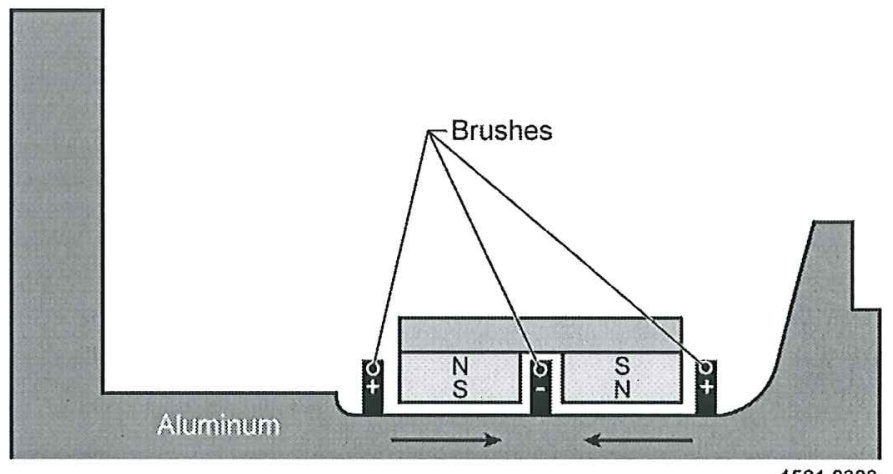

1501.0303

Fig. 19. Electromagnetically actuated filament brushes for motor generator operation.

\section{CONCLUSIONS}

The governing equations for a homopolar null flux bearing have been derived to define the necessary conditions for stable operation. It is possible to combine the tasks of axial stability and damping together into a common component. Temperature calculations have been derived to predict acceptable steady state perturbations from equilibrium. The bearing cannot operate continuously in a vibration-prone environment. Stable operation occurs above $3,400 \mathrm{rpm}$, although modifications to the axial stabilizing magnets might lower this further. Filament brushes present the simplest solution for motor - generator operation.

\section{REFERENCES}

[1] J. Powell and G. Danby, "Electromagnetic Inductive Suspension and Stabilization System for a Ground Vehicle", U.S. Patent \#3,470,828, October 7, 1969.

[2] Y. Kyotani, "Recent progress by JNR on Maglev", IEEE Transactions on Magnetics, vol. MAG-24, no. 2, March 1988, pp. 804-807.

[3] K. Davey, "Analysis of an electrodynamic MAGLEV system," IEEE Transactions on Magnetics, vol. 35, no. 5, pp. 4259-4267, September, 1999.

[4] Kent R. Davey, "Use and analysis of null flux coils," COMPEL International Journal for Computation and Mathematics in Electrical and Electronic Engineering, vol. 22, no. 2, pp. 304-313, March, 2003.

[5] Kent Davey, Tony Morris, Jim Schaaf, and Don Rote, "Calculation of motion induced eddy current forces in null flux coils," IEEE Transactions on Magnetics, vol. 31, no. 6, pp. 4214-4216, 1995.

[6] Kent Davey and Tony Morris, "Passive Magnetic Bearing System for Translation or Rotation", January 2, 1996, U.S. Patent \# 5,481,146.

[7] Paul Alan Basore, "Passive stabilization of flywheel magnetic bearings", MIT-Thesis:1980-21, 1980.

[8] A. V. Filatov and E. H. Maslen, "Passive magnetic bearing for flywheel energy storage systems," IEEE Transactions on Magnetics, 2001, vol. 37, no. 6, pp. 3913-3924.

Fig. 18. Complete bearing with radial, axial, and damping coefficients. 